\title{
Optical Detection of Synaptically Induced Glutamate Transport in Hippocampal Slices
}

\author{
Satoshi Kojima,, ${ }^{1}$ Takeshi Nakamura, ${ }^{1}$ Takahisa Nidaira, ${ }^{3}$ Kyoko Nakamura, ${ }^{2}$ Noriko Ooashi, ${ }^{2}$ Etsuro Ito, ${ }^{1}$ \\ Kei Watase, ${ }^{4}$ Kohichi Tanaka, ${ }^{4}$ Keiji Wada, ${ }^{4}$ Yoshihisa Kudo, ${ }^{2}$ and Hiroyoshi Miyakawa ${ }^{2}$ \\ 1 Division of Biological Sciences, Graduate School of Science, Hokkaido University, Sapporo 060-0810, Japan, \\ ¿2aboratory of Cellular Neurobiology, Tokyo University of Pharmacy and Life Science, Tokyo 192-03, Japan, 3Hamamatsu \\ Photonics K.K., Hamamatsu 812, Japan, and ${ }^{4}$ Department of Degenerative Neurological Diseases, National Institute of \\ Neuroscience, National Center of Neurology and Psychiatry, Kodaira, Tokyo 187-8502, Japan
}

Although it has long been believed that glial cells play a major role in transmitter uptake at synapses in the CNS, the relative contribution of glial and neuronal cells to reuptake of synaptically released glutamate has been unclear. Recent identification of the diverse glutamate transporter subtypes provides an opportunity to examine this issue. To monitor glutamate transporter activity, we optically detected synaptically induced changes of membrane potential from hippocampal CA1 field in slice preparations using a voltage-sensitive dye, $\mathrm{RH} 155$. In the presence of ionotropic glutamate-receptor blockers, synaptic inputs gave rise to a slow depolarizing response (SDR) in the dendritic field. The amplitude of SDR correlated well with presynaptic activities, suggesting that it was related to transmitter release. The SDR was found to be caused by the activities of glutamate transporters because it was not affected by blockers for $\mathrm{GABA}_{\mathrm{A}}$, nACh, 5- $\mathrm{HT}_{3}, \mathrm{P}_{2 \mathrm{x}}$, or metabotropic glutamate receptors but was greatly reduced by dihydrokainate (DHK), a specific blocker for GLT-1 transporter, and by D,L-threo- $\beta$ hydroxyaspartate (THA), a blocker for EAAC, GLAST, and GLT-1 transporters. When SDR was detected with $\mathrm{RH} 482$ dye, which stains both glial and neuronal cells, $1 \mathrm{~mm}$ DHK and $1 \mathrm{~mm}$ THA were equally effective in suppressing SDR. The SDR was very small in GLT-1 knockout mice but was maintained in gerbil hippocampi in which postsynaptic neurons were absent because of ischemia. Because GLT-1 transporters are exclusively expressed in astrocytes, our results provide direct evidence that astrocytes play the dominant role in sequestering synaptically released glutamate.

Key words: glutamate transporter; glutamate uptake; voltagesensitive dye; astrocytes; hippocampus; brain slice
Uptake of glutamate from synaptic clefts in the CNS is important both in terminating signal transmission and in preventing excitotoxicity. It has long been believed that astrocytes play a major role in inactivating neurotransmitters because of their sheet-like shape and location of the highly branched processes that envelope synapses (Kosaka and Hama, 1986) and because of distributions of various enzymes that metabolize transmitters (Martin, 1995). The relative contribution of glial and neuronal uptake of synaptically released glutamate, however, remained uncertain because it was difficult to monitor transporter activities and isolate contributions from glial and neuronal cells.

Recent molecular biological studies have revealed that glutamate transporters can be classified into several subtypes (Malandro and Kilberg, 1996): GLAST (Storck et al., 1992), GLT-1 (Pines et al., 1992), EAAC (Kanai and Hediger, 1992), EAAT4 (Fairman et al., 1995), and EAAT5 (Arriza et al., 1997). They also have different cellular localizations (Rothstein et al., 1994; Lehre et al., 1995; Arriza et al., 1997; Furuta et al., 1997; Nagano et al., 1997). Among these, GLAST and GLT-1 have been shown to be expressed in glial cells, whereas other types are expressed in

Received Nov. 2, 1998; revised Dec. 29, 1998; accepted Jan. 19, 1999.

This work was supported by grants from the Human Frontier Science Program (H.M.) and from the Program for Promotion of Fundamental Studies in Health Science of the Organization of Pharmaceutical Safety and Research (OPSR) Japan (Y.K.). We thank Fred Horvath for helpful comments on this manuscript.

Correspondence should be addressed to Dr. Hiroyoshi Miyakawa, Laboratory of Cellular Neurobiology, Tokyo University of Pharmacy and Life Science, Horinouchi, Hachioji,Tokyo 192-03, Japan.

Copyright (C) 1999 Society for Neuroscience $\quad 0270-6474 / 99 / 192580-09 \$ 05.00 / 0$ neurons or retinal cells. Those findings provide an opportunity to test the old hypothesis of glial dominance in transmitter uptake. Indeed, molecular manipulations of glutamate transporters have suggested a major role of glial uptake. Knockout of GLT-1 glial glutamate transporter gives rise to elevated extracellular glutamate levels, neurodegeneration, and behavioral abnormality (Rothstein et al., 1996; Tanaka et al., 1996), whereas antisense knockdown of EAAC neuronal transporter produced only mild neurotoxicity.

Recent whole-cell studies report direct monitoring of transporter activity from glial cells. Mennerick and Zorumski (1994) reported that an inward current attributable to glutamate uptake at autaptic synapses can be recorded from glial cells in microislands of cultured hippocampi. Clark and Barbour (1997) detected an inward current from cerebellar Bergmann glia cells and supposed it to be caused by glutamate uptake. Bergels and Jahr (1997), Diamond et al. (1998), and Luscher et al. (1998) studied synaptically activated inward current from the cell body of hippocampal astrocytes that was suppressed by blockers of glutamate transporters. Mennerick et al. (1998) recently showed that GLT-1 transporters are functioning in both glial and neuronal cells at autaptic synapses of micro-island cultures. These studies, however, have not examined the question of relative importance of glial and neuronal transporters at normal CNS synapses.

In the present study, we attempted to directly monitor synaptically induced activities of all subtypes of glutamate transporters by monitoring membrane potential with voltage-sensitive dye and a photodiode array system in the CA1 area of hippocampal slice 
preparations. Because all of the presently known glutamate transporters are reported to be electrogenic, one can detect the activity of transporters by monitoring membrane potential. We were able to isolate a depolarizing response that was caused by the activity of glutamate transporters. We examined this response to find that GLT-1 glutamate transporter subtype of astrocytes plays the dominant role in inactivating glutamate released at hippocampal CA1 excitatory synapses.

\section{MATERIALS AND METHODS}

Voltage-sensitive dye measurement from hippocampal slices. Hippocampal slices $(300 \mu \mathrm{m})$ were prepared from adult Wistar rats $(8-12$ weeks old). Slices were maintained in a holding chamber for at least $20 \mathrm{~min}$ and were then stained with voltage-sensitive dye: RH155 (NK3041) or RH482 (NK3630) (Molecular Probes, Eugene, OR, or Nippon Kankoh-Shikiso, Okayama, Japan) $(0.1 \mathrm{mg} / \mathrm{ml}$ for $30 \mathrm{~min})$. For most optical recordings, we used RH155 because it has been reported to stain preferentially glial cells over neuronal cells in skate cerebellum (Konnerth et al., 1987). Stained slices were then placed in an experimental chamber mounted on an inverted microscope (TMD-300, Nikon, Tokyo, Japan). The changes in absorption associated with membrane potential changes were measured with a high-speed (maximum frame rate $2 \mathrm{kHz}$ ) $16 \times 16$ photodiode array system (Agrus-50/PDA, Hamamatsu Photonics, Hamamatsu, Japan). Photo currents generated at photodiodes were converted to voltage with a current-to-voltage converter with $50 \mathrm{M} \Omega$ feed-back resistor, sample-and-holded, and DC-coupled to an analog-to-digital converter that has 16 bit resolution. In most of the experiments, $10 \times$ objective lens (NA 0.45 ) was used. With $10 \times$ objective, each diode imaged a slice area of $52.5 \times 52.5 \mu \mathrm{m}$. Slices were submerged in artificial CSF containing (in mM): $124 \mathrm{NaCl}, 2.5 \mathrm{KCl}, 26 \mathrm{NaHCO}_{3}, 10$ glucose, $1.25 \mathrm{NaH}_{2} \mathrm{PO}_{4}$,

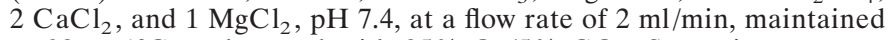
at $32 \pm 1{ }^{\circ} \mathrm{C}$, and gassed with $95 \% \mathrm{O}_{2} / 5 \% \mathrm{CO}_{2}$. Synaptic responses were evoked by delivering a short current pulse of $300 \mu$ sec duration with a bipolar tungsten electrode to Schaffer collaterals. Stimulations were given every $5 \mathrm{sec}$, and the optical responses were averaged over $12-16$ trials. In all experiments, an extracellular field potential recording from stratum pyramidale was simultaneously performed to ensure that the response was consistent. CNQX, APV, MDL72222 (3tropanyl-3,5-dichlorobenzoate), LY278584 (1-methyl- $N$-(8-methyl-8azabicyclo[3,2,1]-oct-3-yl)-1H-indazole-3-carboxamide maleate), MCPG [( \pm$)-\alpha$-methyl-4-carboxyphenylglycine], and t-ACPD were purchased from RBI (Natick, MA). Dihydrokainate (DHK), D, L-threo$\beta$-hydroxyaspartate (THA), bicuculline, and picrotoxin were purchased from Sigma (Milan, Italy). Hexamethonium, suramin, and $d$ tubocurarine were purchased from Wako (Osaka, Japan). CNQX was dissolved in dimethyl sulfoxide (DMSO) and diluted to the superfusion solution with a final concentration for DMSO of $0.1 \%$.

Organotypic slice culture of wild-type and GLT-1 knockout mice hippocampi. Organotypic slice cultures of the hippocampi were prepared from wild-type and GLT-1 knockout mice (Tanaka et al., 1996). Genotype of the mice was determined by PCR analysis applied to genomic DNA isolated from tails. The hippocampi were removed from newborn mice ( $7 \mathrm{~d}$ after birth) and sliced to $300 \mu \mathrm{m}$ thickness with a tissue chopper. Each slice was placed on a filter membrane of a cell-culture insert (Millicel-CM, Millipore, Bedford, MA), and the insert was placed in a well of a culture plate containing $0.6 \mathrm{ml}$ of culture medium (Eagle's minimal essential medium) supplemented with $25 \%$ heat-inactivated horse serum. The slices were cultured for 14-18 d before use.

Gerbil hippocampal slices after brief ischemia. Mongolian gerbils (male, 12-14 weeks old) were anesthetized with $2.0 \%$ halothane, $30 \% \mathrm{O}_{2}$, and $68 \% \mathrm{~N}_{2} \mathrm{O}$. Common carotid arteries were bilaterally clamped with surgical clips for $5 \mathrm{~min}$. Rectal temperature was maintained at $37.5-38.0^{\circ} \mathrm{C}$ throughout the procedure (Kirino, 1993). After recovery from anesthesia, gerbils were housed in cages with free access to water and pellet food. Seven days after ischemia, slices were made from the left hippocampi. To confirm that CA1 pyramidal cells had degenerated, the right hemispheres were fixed by $4 \%$ paraformaldehyde/PBS, serially sectioned at $10 \mu \mathrm{m}$ on a microtome, and stained with cresyl violet.

\section{RESULTS}

\section{Slow depolarizing response}

To directly examine the activity of synaptically induced glutamate transporters at excitatory synapses in mature CNS, we optically monitored synaptically evoked changes of membrane potential in the hippocampal CA1 area. Rat hippocampal slices were stained with voltage-sensitive dye, RH155 (NK3041) or RH482 (NK3630), and the changes in absorption were measured with a $16 \times 16$ photodiode array at a $2 \mathrm{kHz}$ frame rate. In response to Schaffer collateral stimulation, depolarizing responses with several components were detected from various laminar in the CA1 field (Fig. 1 $A, B$ ). In stratum radiatum, a sharp depolarizing response was followed by a large depolarization that decayed in several tens of milliseconds. In stratum pyramidale, fast depolarizing responses were detected in addition to slower depolarization. Blockage of ionotropic glutamatergic receptors, by applying CNQX $(10 \mu \mathrm{M})$ and APV $(50 \mu \mathrm{M})$ or using kynurenic acid (5 $\mathrm{mM})$, abolished fast responses in the stratum pyramidale and suppressed a large portion of the slower depolarizing component in both stratum radiatum and stratum pyramidale. Under these conditions, optical responses were detected only from the stratum radiatum: a fast depolarization and a slow depolarizing response (SDR). The SDR under these conditions (hereafter we call this component SDR) peaked much later than the response under normal conditions. In this condition, extracellularly recorded population spikes and field EPSPs were completely abolished, leaving only presynaptic fiber volley component in stratum radiatum (Fig. 1B).

The SDR was blocked in $\mathrm{Ca}^{2+}$-free medium (Fig. $1 A, B$ ) and by $0.1 \mathrm{mM} \mathrm{Cd}^{2+}$ (data not shown), suggesting that it was related to transmitter release. The fast depolarizing response left in $\mathrm{Ca}^{2+}$. free medium is caused by the activity of presynaptic fiber volleys, as confirmed by the fact that it was blocked by adding $1 \mu \mathrm{M}$ TTX in parallel with the blockade of extracellularly recorded presynaptic fiber volley. In $\mathrm{Ca}^{2+}$-free medium, a small residual depolarization, which was also blocked with TTX, was detected, but we made no attempt to characterize this component.

To confirm that SDR was really a membrane depolarization and not an intrinsic signal such as changes in light scattering, we monitored the response at two different wavelengths. Because of the optical properties of the dye, both dyes should show an increase in absorption at $700 \mathrm{~nm}$ and a decrease in absorption at $610 \mathrm{~nm}$ (Konnerth et al., 1987) on depolarization. With both RH155 (Fig. 1C) and RH482 (data not shown), the absorption increased at $700 \mathrm{~nm}$ and decreased at $610 \mathrm{~nm}$, thus indicating that the SDR was indeed a membrane depolarization.

To characterize SDR, the spatial distribution of SDR was compared with that of postsynaptic responses and presynaptic activities. Glutamatergic postsynaptic response (Fig. $2 A$, iv) was isolated by subtracting responses in CNQX/APV (Fig. $2 A$, ii) from those under normal conditions (Fig. $2 A, i$ ). SDR (Fig. $2 A, v$ ) was isolated by subtracting responses in $\mathrm{Ca}^{2+}$-free medium (Fig. $2 A$, iii) from those in CNQX/APV medium (Fig. $2 A$, ii). The amplitude of SDR correlated very well (correlation coefficient $=$ 0.96) with that of presynaptic fiber volley (Fig. 2B). Postsynaptic responses were detected from all over the dendritic layer and the cell body layer and showed poor correlation with presynaptic fiber volley (Fig. 2B). Strong correlation of SDR with presynaptic activity despite its $\mathrm{Ca}^{2+}$ sensitivity suggests that SDR is related to transmitter release.

\section{Pharmacological characterization of SDR}

This SDR was not significantly suppressed (data not shown) by the blockers of $\mathrm{GABA}_{\mathrm{A}}$ (bicuculline $10 \mu \mathrm{M}$, picrotoxin $10 \mu \mathrm{M}$ ), nACh [hexamethonium chloride $100 \mu \mathrm{M}, d$-tubocurarine $2 \mu \mathrm{M}$ ),

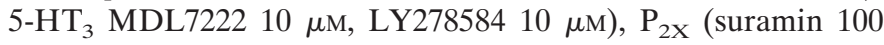



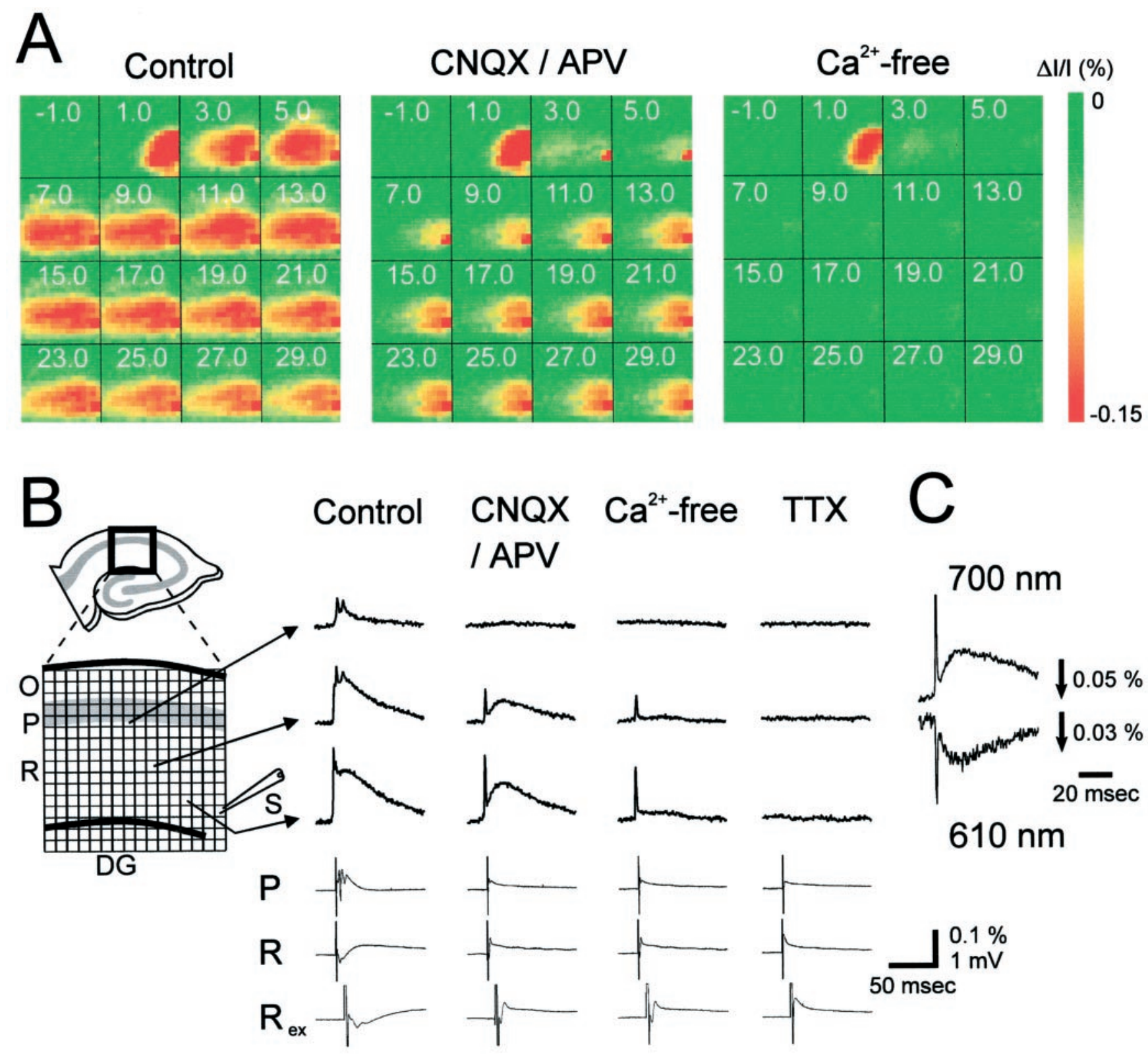

Figure 1. SDR in stratum radiatum of the hippocampal CA1 region. $A$, Pseudo-color image sequences of synaptically induced changes of membrane potentials in three different conditions. Red indicates depolarization; green indicates hyperpolarization. Change in absorption at each pixel was monitored at $700 \mathrm{~nm}$ and normalized to the total light intensity at respective pixels. Voltage-sensitive dye: RH155. Frame rate: $2 \mathrm{kHz}$. Every fourth frame (2 msec intervals) is shown. Numbers in the frames indicate elapsed times (milliseconds) from the electrical stimulation delivered at stratum radiatum. Images were taken from the area $(840 \times 840 \mu \mathrm{m})$ schematically drawn in B. Left, In normal medium; middle, in CNQX $(10 \mu \mathrm{M})$ and APV $(50 \mu \mathrm{M})$; right, in $\mathrm{Ca}^{2+}$-free medium. $B$, Time course of optical signals recorded from three elements of the photodiode array under the same three conditions as shown in $A$ plus TTX. Top denotes depolarization. Shown below are the extracellular field potentials recorded from stratum pyramidale $(P)$ and stratum radiatum $(R)$ in the same conditions shown on the same time scale. The potentials recorded from stratum radiatum $(R)$ are expanded three times and shown at the bottom $\left(R_{e x}\right)$. CNQX $(10 \mu \mathrm{M}) / \mathrm{APV}(50 \mu \mathrm{M})$ blocked the optical responses in stratum pyramidale, leaving a fast depolarizing component corresponding to presynaptic fiber volley and an SDR in stratum radiatum. In this condition, the extracellular recording showed only a presynaptic fiber volley component. The SDR was abolished in $\mathrm{Ca}^{2+}$-free medium, and all responses were blocked by TTX $(1 \mu \mathrm{M})$. $O$, Stratum orience; $P$, stratum pyramidale; $R$, stratum radiatum; $D G$, dentate gyrus; $S$, stimulating electrode. $C$, SDR detected with RH155 dye at two different wavelengths: 700 and $610 \mathrm{~nm}$.

$\mu \mathrm{M}$ ), or metabotropic glutamate receptors (MCPG $0.3 \mathrm{~mm}$ ). These results indicate that SDR is unlikely to be caused by activation of postsynaptic receptors. SDR was detected from cultured slice preparations as well (data not shown), suggesting that SDR is unlikely to be caused by modulatory inputs such as cholinergic, aminergic, or peptidergic inputs from other regions of the brain.
The SDR monitored with RH155 was reversibly suppressed (Fig. $3 B$ ) by $1 \mathrm{~mm}$ DHK, a glutamate transporter blocker $(51.6 \pm$ $3.3 \%$ in DHK, $n=10$; all of the statistical values are mean $\pm \mathrm{SE}$ ), which demonstrates that SDR is mainly caused by activation of glutamate transporters. Glutamate transporters are known to be expressed in both neuronal and glial cells. At a concentration of $<3 \mathrm{~mm}$, DHK is reported to specifically block a GLT-1 subtype of 
A

\section{(i) Control}

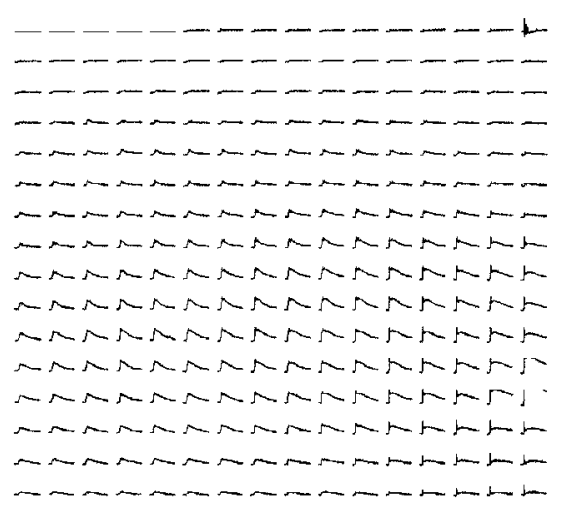

(ii) CNQX / APV

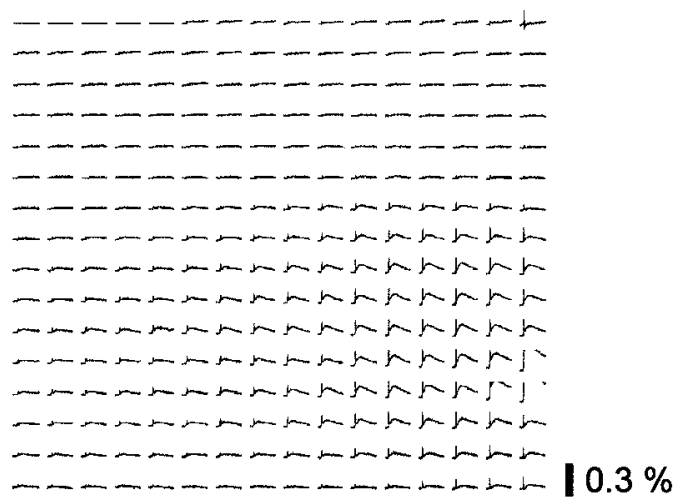

(iii) Presynaptic Fiber Volley

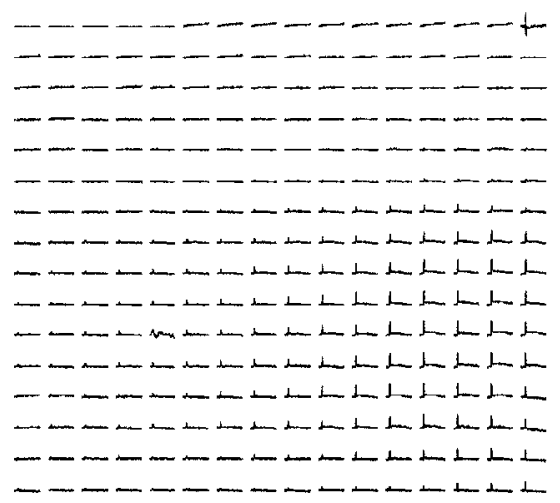

(iv) Postsynaptic Response

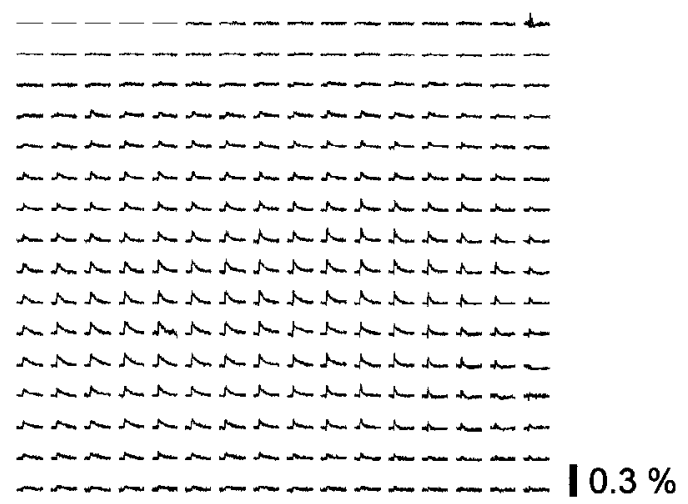

\section{(v) Slow Depolarizing Response}
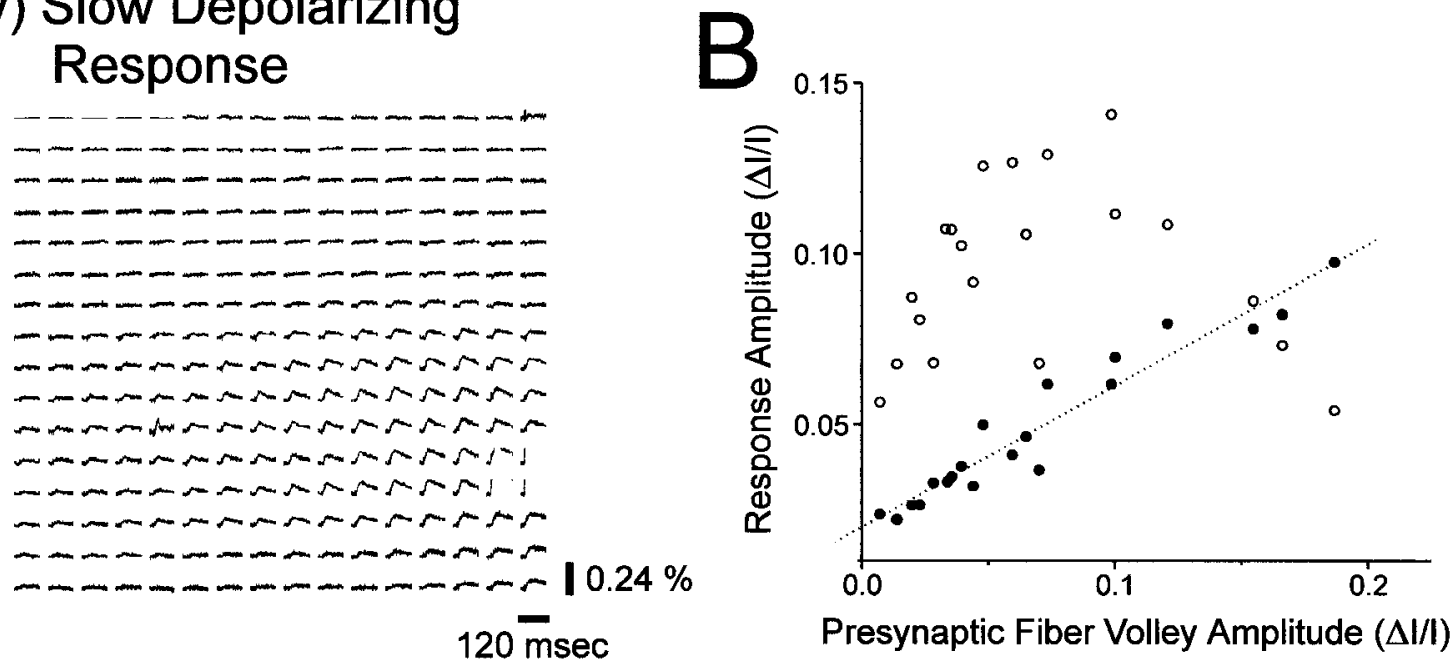

Figure 2. Spatial profile of SDR. $A$, Spatial profiles of $(i)$ control response in normal medium, (ii) response in media containing CNQX (10 $\mu \mathrm{M})$ and APV $(50 \mu \mathrm{M}),\left(\right.$ iii) presynaptic fiber volley, (iv) postsynaptic response, and (v) SDR. Presynaptic fiber volley was recorded in Ca ${ }^{2+}$-free medium. Postsynaptic response is a CNQX/APV-sensitive component obtained by subtracting responses in CNQX/APV from the control. SDR was obtained by subtracting responses in $\mathrm{Ca}^{2+}$-free medium from those in CNQX/APV-containing medium. $B$, Correlation of the amplitudes of SDR and presynaptic fiber volley. Abscissa: the amplitude of presynaptic fiber volley. Ordinate: the amplitude of responses. $\bullet$, SDR; $\bigcirc$, postsynaptic response. Dotted line is the regression line for SDR amplitude and presynaptic fiber volley amplitude (correlation coefficient $=0.96$ ). 
A<smiles></smiles>

$\mathrm{RH} 155$
$0.1 \%$

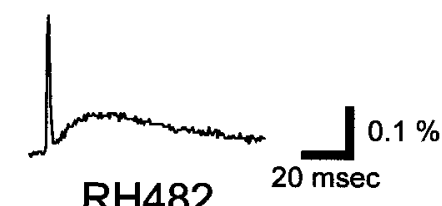

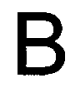

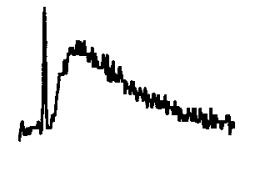

CNQX / APV
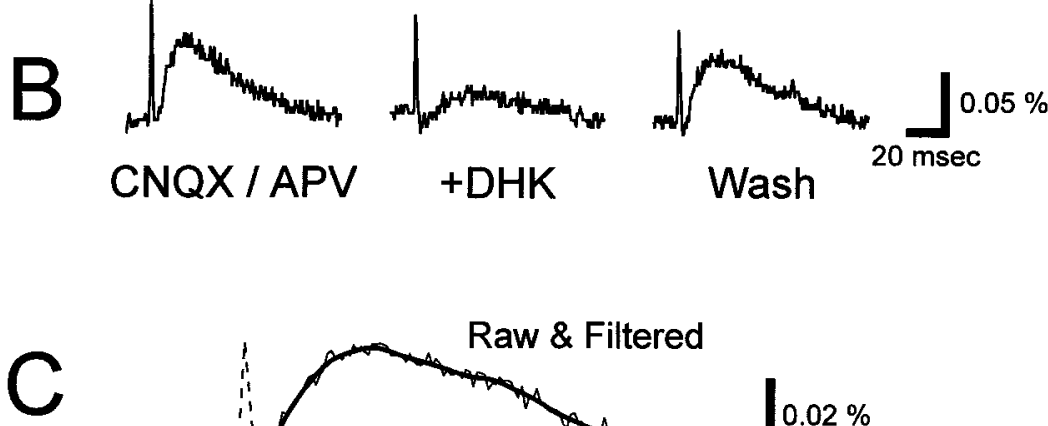

Figure 3. Glutamate transporter activities give rise to SDR. A, Representative SDRs from RH155- and RH482stained slices. The relative amplitude of SDR to the presynaptic fiber volley measured with RH155 $(0.78 \pm 0.10$, $n=12$ ) was significantly $(p<0.01)$ greater than that with RH482 (0.46 $\pm 0.02, n=12) . B$, SDR in RH155-stained slices was suppressed by $1 \mathrm{~mm} \mathrm{DHK}(51.6 \pm 3.3 \%, n=10)$. $C$, The DHK-sensitive component in the RH155-stained slice was isolated by subtracting responses in DHK + CNQX/APV medium from the responses in CNQX/APVcontaining medium. Traces from 24 pixels in stratum radiatum were averaged (thin line) and smoothed (thick line) by an FFT filter with a cutoff frequency of $333 \mathrm{~Hz}$. The mean time-to-peak of SDR from the onset of presynaptic fiber volley (dotted line) was $16.2 \pm 1.6 \mathrm{msec}(n=8)$, with a $20-80 \%$ rise time of $5.56 \pm 0.41 \mathrm{msec}(n=8)$. The half-decay time was $27.6 \pm 4.3 \mathrm{msec}(n=8) . D$, SDRs in RH482-stained slices were suppressed by $1 \mathrm{~mm}$ DHK $(58.0 \pm 3.9 \%, n=6)$ or 1 mM THA $(50.4 \pm 2.9 \%, n=5)$ and to a similar extent. Five repetitive stimuli were given at $100 \mathrm{~Hz}$.

glutamate transporter that is localized only in astrocytes (Rothstein et al., 1994) without suppressing synaptic transmission. Because agonists of metabotropic glutamate transporters only slightly suppressed SDR (data not shown), it is not likely that this suppressive effect of DHK is attributable to presynaptic inhibition of transmitter release caused by enhanced activation of metabotropic glutamate transporters in the presynaptic terminals.

To measure the time course of SDR, the DHK-sensitive component was isolated by subtracting responses in DHK + CNQX/ APV medium from the responses in CNQX/APV medium and smoothed by a fast Fourier transform (FFT) filter with a cutoff frequency of $333 \mathrm{~Hz}$ (Fig. 3C). The mean time-to-peak of SDR from the onset of presynaptic fiber volley was $16.2 \pm 1.6 \mathrm{msec}$ $(n=8)$, with a $20-80 \%$ rise time of $5.56 \pm 0.41 \mathrm{msec}(n=8)$. The half-decay time was $27.6 \pm 4.3 \mathrm{msec}(n=8)$.

To estimate the relative contribution of glial and neuronal glutamate transporters to SDR, we stained slices with RH482, evoked SDR by delivering five repetitive inputs at $100 \mathrm{~Hz}$, and compared (Fig. 3D) the effects of DHK and another transporter blocker, THA, which blocks both neuronal (EAAC) and glial (GLT-1 and GLAST) transporters with similar potency (Arriza et al., 1994). When Schaffer collaterals were stimulated repeat-

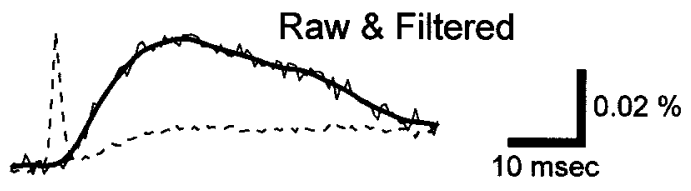

\section{DHK-sensitive Component}
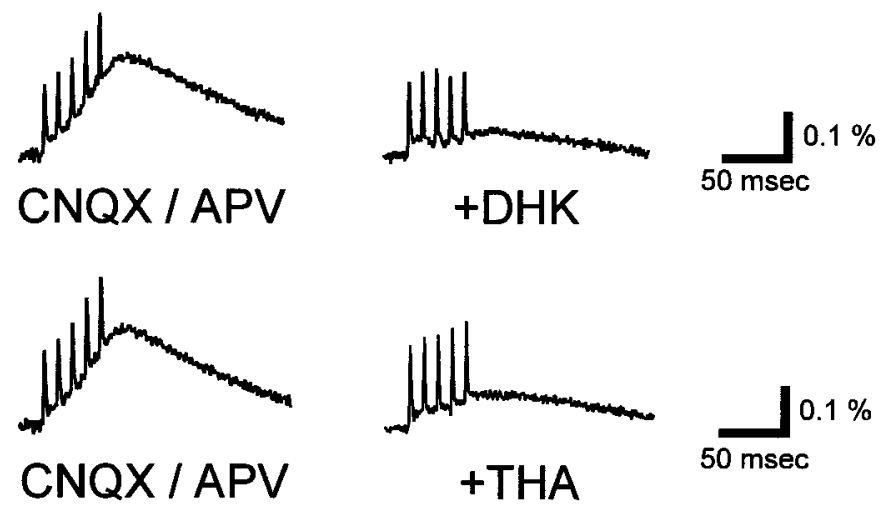

$0.1 \%$

edly with 10 msec intervals, the amplitude of SDR was constant in response to each of the first four to five stimuli, after which the overall depolarization reached a plateau level. Both DHK and THA have been shown to have a similar $K_{\mathrm{i}}$ value $(23 \pm 6 \mu \mathrm{M}$ and $19 \pm 6 \mu \mathrm{M}$, respectively) to suppress GLT-1-type transporter with similar potency $(>95 \%$ suppression at $1 \mathrm{~mm})$ when tested on transporters transfected in COS cells (Arriza et al., 1994). We chose RH482 dye for this particular experiment because RH482 stains both neuronal and glial cells in contrast to RH155, which is reported to stain glial cells preferentially over neuronal cells (Konnerth et al., 1987). The relative amplitude of SDR to presynaptic fiber volley was significantly $(p<0.01)$ greater with RH155 $(0.78 \pm 0.10, n=12)$ than with RH482 $(0.46 \pm 0.02, n=$ 12). This fact by itself shows that the contribution to SDR of glial cells is greater than that of neuronal cells.

If neuronal transporter is participating in SDR, then the extent to which THA blocks SDR should be greater than that of DHK. The suppression of SDR in $1 \mathrm{~mm}$ DHK $(58.0 \pm 3.9 \%, n=6)$ and 1 mм THA $(50.4 \pm 2.9 \%, n=5)$ was similar and not significantly different at the $5 \%$ level. When $1 \mathrm{~mm}$ THA was applied in addition to $1 \mathrm{~mm}$ DHK, SDR was greatly suppressed (83.2 \pm $4.4 \%, n=6$ ). At a higher dose of $3 \mathrm{~mm}$, DHK almost completely 
A

Control +CNQX/APV +CNQX/APV

Wild-type<smiles>C=CCCCC</smiles>

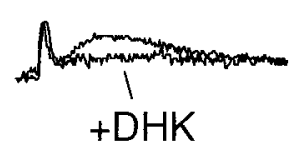

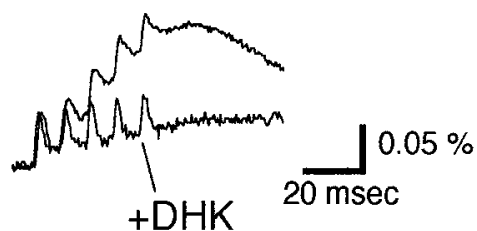

GLT-1
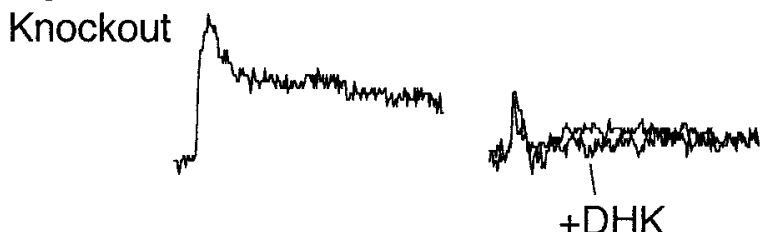

B

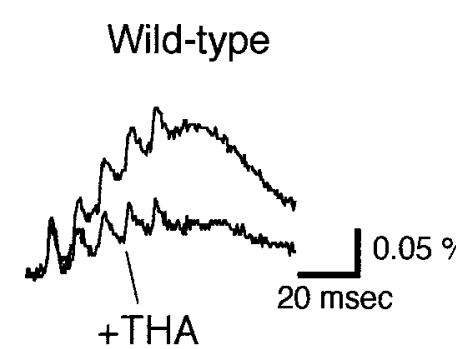

GLT-1
Knockout

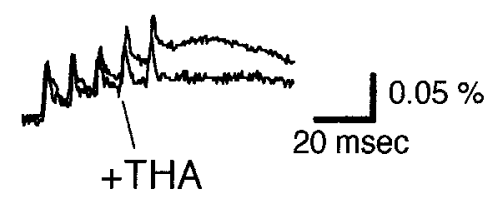

C

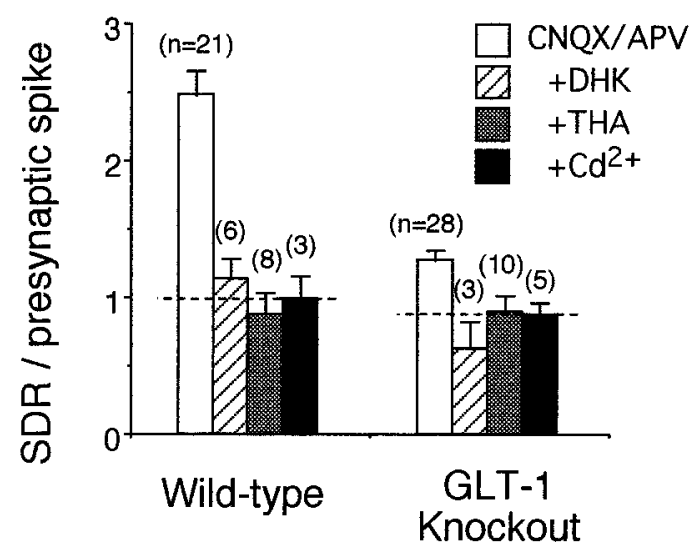

$0.025 \%$ Figure 4. Small SDRs in GLT-1 $20 \mathrm{msec}$ + DHK SDR in stratum radiatum of organotypic hippocampal slice cultures made from wild-type and GLT-1 knockout mice. Left traces, Optical responses to stratum radiatum stimulation. Middle traces, SDR in medium containing CNQX $(10 \mu \mathrm{M}) / \mathrm{APV}(50 \mu \mathrm{M}) ; 1 \mathrm{~mm}$ DHK blocked most of the SDRs in wildtype mice. Right traces, SDR for five repetitive inputs $(100 \mathrm{~Hz})$. Relative amplitude of SDR was smaller in knockout. $B$, Effect of THA on GLT-1 knockout mice. $C$, Comparison of SDR in various conditions. The amplitude of evoked depolarization induced by five repetitive inputs given at $100 \mathrm{~Hz}$ was normalized to the amplitude of the presynaptic fiber volley component. White column: In CNQX (10 $\mu \mathrm{M}) / \mathrm{APV}(50$ $\mu \mathrm{M})$. Relative amplitude of depolarization was $2.48 \pm 0.18(n=21)$ for wildtype mice and $1.27 \pm 0.07(n=28)$ for GLT-1 knockout mice. Hatched column: With 1 mM DHK in addition to CNQX/ APV. Shaded column: With 1 mM THA in addition to CNQX/APV. DHK or THA suppressed most of the depolarizing response in wild-type mice. In knockout mice, the extent of suppression was small. Black column: In $1 \mathrm{~mm}$ $\mathrm{Cd}^{2+}$. A Cd ${ }^{2+}$-insensitive nonsynaptic component was detected in both wildtype $(0.99 \pm 0.17, n=3)$ and knockout mice $(0.87 \pm 0.09, n=5)$. The large part of the depolarization of knockout mice in the presence CNQX/APV was this $\mathrm{Cd}^{2+}$-insensitive component. suppressed SDR $(88.3 \pm 1.9 \%, n=4)$. Although it has been reported that DHK and THA almost completely suppress transporter that was transfected in COS cells (Arriza et al., 1994), it is conceivable that it takes a higher dose in slice preparations because of poor penetration of the drugs into the tissue. Because THA has been reported to have similar potency to suppress GLT-1, GLAST, and EAAC (Arriza et al., 1994), and both DHK and THA show similar potency for GLT-1, these results suggest that the contribution of GLT-1 subtype of the glutamate transporter is greater than that of GLAST or EAAC subtypes.

\section{SDR in organotypic cultured slice prepared from GLT-1 knockout mice}

To confirm that the SDR is caused by the activity of GLT-1 glutamate transporter subtype, we prepared hippocampal slices from newborn GLT-1 knockout mice and organotypically cultured the slices for 2-3 weeks. GLT-1 knockout preparation showed normal synaptic responses that consisted of presynaptic fiber volley, CNQX/APV-sensitive EPSP, and small SDR as shown in Figure $4 A$. In response to the same intensity of stimulation, presynaptic fiber volley with similar amplitude and time course was evoked. To compare amplitude of SDR in wild-type and knockout preparations, from the array covering stratum radiatum we selected four (or eight) pixels that were $200 \mu \mathrm{m}$ away from the stimulating electrode and calculated SDR/fiber volley ratio by dividing the averaged amplitude of SDR by the amplitude of presynaptic fiber volley. We found that relative amplitude of synaptically induced DHK/THA-sensitive depolarization was very small in GLT-1 knockout mice (Fig. 4). This finding implies 


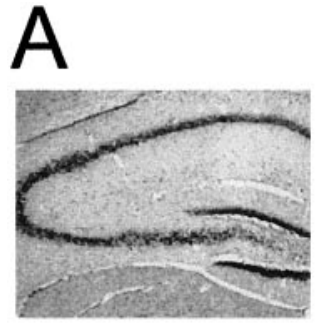

\section{Normal}

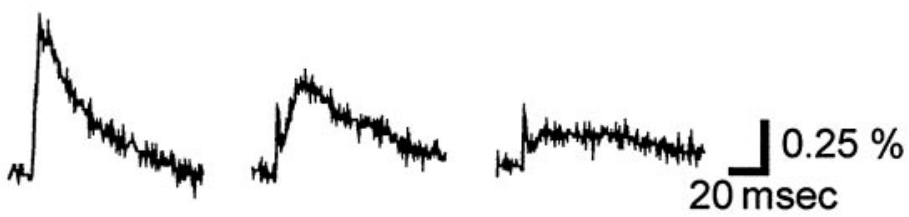

\section{After Ischemia}

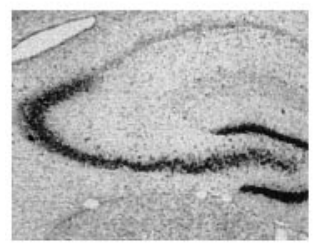

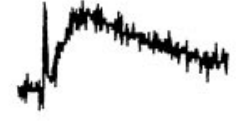

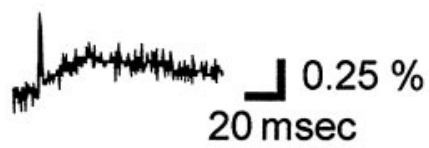
( $n=7)$ and ischemic $(58.6 \pm 2.3 \%, n=$ 6) preparations.

\section{CNQX I APV \\ + DHK \\ Control}

$$
\text { APV }
$$

that the contribution of GLT-1 is dominant among other transporter subtypes in generating SDR.

Repetitive simulation revealed a small but significant slow depolarizing response in GLT-1 knockout mice (Fig. 4). This small SDR was sensitive to DHK (Fig. $4 A$ ), and the extent of blockade was similar to that by THA or $\mathrm{Cd}^{2+}$ (Fig. 4B,C). This residual SDR in knockout mice could be attributable to the activity of novel DHK-sensitive glutamate transporters, which are overexpressed in knockout animals.

\section{SDR in hippocampi with ischemic damage}

To confirm further that SDR is of glial origin, we tested to determine whether SDR can be measured from preparations with no postsynaptic neurons (Fig. 5). SDR was evoked in the CA1 dendritic field of gerbil hippocampal slices made $7 \mathrm{~d}$ after $5 \mathrm{~min}$ of ischemia (Fig. 5B). In this preparation, glial cells and Shaffer collaterals are intact but CA1 pyramidal neurons are absent (Kirino, 1993). In a normal medium, the time course of synaptically evoked response recorded from stratum radiatum of normal gerbils is similar to that of rats: a sharp depolarizing response followed by large, slower depolarization (Fig. 5A). Application of $10 \mu \mathrm{M}$ CNQX and $50 \mu \mathrm{M}$ APV blocked the fast EPSP component and revealed DHK-sensitive SDR in normal slices. In hippocampal slices with ischemic damage, synaptically evoked responses in normal medium recorded from stratum radiatum showed a fast presynaptic component and SDR, but they lacked the large EPSP component, as shown by the fact that CNQX/APV application did not significantly suppress the synaptically induced response (Fig. 5B). The SDR/fiber volley ratio in ischemic gerbil slices $(0.86 \pm 0.075, n=11)$ was not significantly different at the $5 \%$ level from that in control slices $(0.929 \pm 0.081, n=5)$. The extent of suppression by DHK (1 mM) was similar in both normal (68.3 \pm $4.8 \%, n=7)$ and ischemic $(58.6 \pm 2.3 \%, n=6)$ preparations. These results show that postsynaptic neurons do not contribute significantly to SDR. We thus concluded that SDR is caused by activities of glial cells and not neuronal cells.

\section{DISCUSSION}

\section{Dominant role of glial GLT-1 transporters in glutamate uptake}

Optical measurement of membrane potential at hippocampal synapses enabled us to directly monitor synaptically evoked activity of glutamate transporters that had been prevented by the morphological complexity of those synapses. Although whole-cell recordings from glial cells have recently been used to study glial transporter activities, it is only with optical measurement that the relative importance of glial transporters and neuronal transporters can be compared, and the time course of transporter activities can be tracked without being distorted by electrical filtering in glial fine processes. We have concluded that the slow depolarizing response revealed after blockade of ionotropic glutamate transmission is attributable to activity of GLT-1 glutamate transporter subtype in astrocytes, on the basis of the following findings: (1) SDR is $\mathrm{Ca}^{2+}$ dependent; (2) SDR correlates well with presynaptic activity; (3) SDR is resistant to blockers of metabotropic glutamate receptors; (4) SDR is resistant to blockers of $\mathrm{GABA}_{\mathrm{A}}$, nACh, 5- $\mathrm{HT}_{3}$, and $\mathrm{P}_{2 \mathrm{X}}$ receptors; (5) SDR can be blocked by a selective blocker of glial glutamate transporter, DHK; (6) SDR detected with RH155 is larger than that detected with RH482; (7) SDR detected with RH482 is equally sensitive to $1 \mathrm{mM}$ DHK and 1 mM THA; (8) SDR is very small in knockout of GLT-1, which is reported to be specifically expressed in astrocytes; and (9) SDR is maintained in preparation with no postsynaptic neurons.

In fish cerebellar slices, Konnerth et al. (1987) optically detected a synaptically induced delayed depolarization that reached its peak in 150-200 msec and was blocked by $\mathrm{Cd}^{2+}$. They attributed this depolarization to accumulation of $\mathrm{K}^{+}$ions that pass through $\mathrm{Ca}^{2+}$-dependent $\mathrm{K}^{+}$channels. It is possible that this depolarization was also caused by the activity of transmitter transporters in glial cells. The slow, $3 \mathrm{sec}$ coupling constant of their recording system or the lower temperature of $22-25^{\circ} \mathrm{C}$ may account for the slower time course of their signal. In rat hippocampal slices, Barish et al. (1996) optically detected a synap- 
tically evoked delayed depolarization in 4-AP-containing medium using RH155 dye. Although their conclusion was that this depolarization was caused by accumulation of $\mathrm{K}^{+}$ions, it may be that they detected the SDR signal we report in this study.

It is of no surprise that the activity of glutamate transporters gives rise to depolarization of glial cells. It has been reported that the glutamate transporters are electrogenic (Bouvier et al., 1992; Arriza et al., 1994; Kanai et al., 1995; Klockner et al., 1995; Zerangue and Kavanaugh, 1996), with net inward current and outward $\mathrm{K}^{+}$current coupled to inward glutamate transport. Indeed, synaptically induced transporter current was recorded at autapses in cultured single neuron micro-islands (Mennerick and Zorumski, 1994) and from Bergmann cells (Clark and Barbour, 1997) and astrocytes in slice preparations (Bergles and Jahr, 1997). Because the input resistance of astrocytes in the hippocampal CA1 area is reported to be fairly high, $\sim 80 \mathrm{M} \Omega$ (McKhann et al., 1997; D'Ambrosio et al., 1998), it is reasonable to expect that the net inward current attributable to activation of glutamate transporters would give rise to substantial depolarization. Because of the spherical profile of astrocyte processes and the random and even distribution of astrocytes in the CA1 field, transporter current generated at various locations of different astrocytes would be cancelled out and could not be detected as extracellular field potential. The depolarization could be attributable in part to accumulation of $\mathrm{K}^{+}$ions in extracellular space because GLT-1 transporters pump out $\mathrm{K}^{+}$ions and change the reversal potential for $\mathrm{K}^{+}$ions. The residual depolarization in $\mathrm{Ca}^{2+}$-free medium may also be caused by the accumulation of $\mathrm{K}^{+}$ions in extracellular space attributable to the activity of presynaptic fibers and terminals.

Because all of the known glutamate transporter subtypes are reported to be electrogenic, our optical measurement of membrane potential should detect activities of all types of glutamate transporters. Our finding that SDR is caused by the activity of astrocytic GLT-1 glutamate transporters implies that astrocytes play the dominant role in sequestering glutamate, which had been suggested by other experimental evidence. If there are unknown types of neuronal glutamate transporters that are not electrogenic, or the electrogeniticity of GLT-1 is much greater than that of others, it is possible that nonglial uptake makes an important contribution. The residual DHK-sensitive component of SDR in GLT-1 knockout mice suggests that there might be novel types of glutamate transporter that can be overexpressed in knockout animals.

Although our results directly showed that uptake of synaptically released glutamate is not caused by activity of transporters in postsynaptic neurons, possible contribution of presynaptic transporters has not been directly ruled out. Our conclusion of a dominant role of astrocytes on glutamate uptake relies on immunocytochemical studies (Rothstein et al., 1994; Lehre et al., 1995) that showed exclusive expression of GLT-1 transporters on glial cells. If GLT-1 is functioning in presynaptic terminals, it is possible that glutamates are sequestered by both astrocytes and presynaptic terminals. It has been reported, however, that immunoprecipitations of not only GLT-1 but also of EAAC and GLAST were not found in presynaptic terminals in hippocampus (Rothstein et al., 1994).

\section{Implications for synaptic transmission}

Because the SDR turned out to be caused by the activities of glutamate transporters, the time course of SDR should give us information about the time course of glutamate concentration at the synaptic cleft. It has been shown that the membrane potentials of hippocampal astrocytes change with short time constants, a few milliseconds or less, in response to current injection (Bergles and Jahr, 1997; D'Ambrosio et al., 1998). Under such conditions, the transporter current should be mostly resistive, and the time course of the membrane potential reflects the time course of transporter currents. Indeed, Bergles and Jahr (1997) reported that the time course of synaptically induced glial response was the same whether it was recorded in current-clamp or voltage-clamp mode. The time course of SDR should then be the same as the time course of transporter activity.

If transporters are not saturated with glutamate, the time course of transporter activity reflects the time course of glutamate molecules available for transporters. The amount of glutamate available for the transporters, however, depends on many factors, such as the volume and morphology of the synaptic cleft and the kinetics and density distribution of the glutamate receptors and transporters (Hestrin et al., 1990; Lester et al., 1990; Clements et al., 1992; Sarantis et al., 1993; Mennerick and Zorumski, 1995; Lester et al., 1996; Barbour and Hausser, 1997; Diamond and Jahr, 1997). It is very likely that the rise and decay time of glutamate concentration near the release sites or receptor sites is much faster than that near the transporters. The time course of SDR then gives us a slower estimate for the rise and decay time course of glutamate at the synaptic cleft. To more accurately estimate the concentration of glutamate available for postsynaptic receptors, model calculation is necessary (Clements et al., 1992; Clements, 1996; Diamond and Jahr, 1997). Our findings will provide important constraints in the understanding of the processes taking place at the synaptic cleft.

The rise and decay times of SDR in the present study are comparable to those reported in previous whole-cell studies that recorded transporter currents from the cell bodies of astrocytes in various preparations. Bergles and Jahr (1994) reported that in the CA1 field of hippocampal slice, the synaptically induced transporter current rises quickly with a $20-80 \%$ rise time of $3.7 \pm 3.1$ msec and decays with a half-decay time of $17.8 \pm 3.1 \mathrm{msec}$. In cerebellar Bergmann glia cells, Clark and Barbour (1997) reported a $20-80 \%$ rise time of $2.36 \pm 0.7 \mathrm{msec}$ and a decay time constant of $25 \pm 6$ msec. Mennerick and Zorumski (1994) have shown a longer decay time constant $(43.1 \pm 2.3 \mathrm{msec})$ of glial response in micro-islands of cultured hippocampi. Although the half-decay time of SDR $(27.6 \pm 4.3 \mathrm{msec})$ is similar to those values, the rise time of $\operatorname{SDR}(5.56 \pm 0.41 \mathrm{msec})$ is longer than those measured with whole-cell recordings. This is puzzling because the higher temperature in our condition should make the transporter activity faster, and the voltage-sensitive dye should detect membrane potentials directly from fine processes where uptake is taking place. One possible explanation is that our method detects transporter activities from different populations of cells or locations of the cells in addition to those detected with the whole-cell recording techniques.

Kinetic analysis of EAAT2 (the human counterpart of GLT-1) has shown that one cycle of transporter action takes $\sim 70 \mathrm{msec}$ (Wadiche et al., 1995). Despite such a slow turnover rate, it has been reported that glutamate transporters determine the time course of synaptic transmission at synapses onto cerebellar Purkinje neurons (Barbour et al., 1994) and also at calyceal synapses in the nucleus magnocellularis (Otis et al., 1996). At Schaffer collateral synapses onto hippocampal CA1 pyramidal neurons, it has been shown that transporters are not involved in terminating synaptic transmission under normal conditions (Hestrin et al., 
1990; Isaacson and Nicoll, 1993; Sarantis et al., 1993). A careful isolation of non-NMDA receptor-mediated and NMDA receptor-mediated EPSPs would allow us to compare the time course of EPSPs and that of transporter activities.

\section{REFERENCES}

Arriza JL, Fairman WA, Wadiche JI, Murdoch GH, Kavanaugh MP, Amara SG (1994) Functional comparisons of three glutamate transporter subtypes cloned from human motor cortex. J Neurosci 14:5559-5569.

Arriza JL, Eliasof S, Kavanaugh MP, Amara SG (1997) Excitatory amino acid transporter 5, a retinal glutamate transporter coupled to a chloride conductance. Proc Natl Acad Sci USA 94:4155-4160.

Barbour B, Hausser M (1997) Intersynaptic diffusion of neurotransmitter. Trends Neurosci 20:377-384.

Barbour B, Keller BU, Llano I, Marty A (1994) Prolonged presence of glutamate during excitatory synaptic transmission to cerebellar Purkinje cells. Neuron 12:1331-1343.

Barish ME, Ichikawa M, Tominaga T, Matsumoto G, Iijima T (1996) Enhanced fast synaptic transmission and a delayed depolarization induced by transient potassium current blockade in rat hippocampal slice as studied by optical recording. J Neurosci 16:5672-5687.

Bergles DE, Jahr CE (1997) Synaptic activation of glutamate transporters in hippocampal astrocytes. Neuron 19:1297-1308.

Bouvier M, Szatkowski M, Amato A, Attwell D (1992) The glial cell glutamate uptake carrier countertransports $\mathrm{pH}$-changing anions. Nature 360:471-474.

Clark BA, Barbour B (1997) Currents evoked in Bergmann glial cells by parallel fibre stimulation in rat cerebellar slices. J Physiol (Lond) 502.2:335-350.

Clements JD (1996) Transmitter timecourse in the synaptic cleft: its role in central synaptic function. Trends Neurosci 19:163-171.

Clements JD, Lester RAJ, Tong G, Jahr C, Westbrook GL (1992) The time course of glutamate in the synaptic cleft. Science 258:1498-1501.

D’Ambrosio R, Wenzel J, Schwartzkroin PA, McKhann II GM, Janigro D (1998) Functional specialization and topographic segregation of hippocampal astrocytes. J Neurosci 18:4425-4438.

Diamond JS, Jahr CE (1997) Transporters buffer synaptically released glutamate on a submillisecond time scale. J Neurosci 17:4672-4687.

Diamond JS, Bergles DE, Jahr CE (1998) Glutamate release monitored with astrocytes transporter currents during LTP. Neuron 21:425-433.

Fairman WA, Vandenberg RJ, Arriza,JL, Kavanaugh MP, Amara SG (1995) An excitatory-acid transporter with properties of a ligand-gated chloride channel. Nature 375:599-603.

Furuta A, Rothstein JD, Martin LJ (1997) Glutamate transporter protein subtypes are expressed differentially during Rat CNS development. J Neurosci 17:8363-8375.

Hestrin S, Sah P, Nicoll RA (1990) Mechanisms generating the time course of dual component excitatory synaptic currents recorded in hippocampal slices. Neuron 5:247-253.

Isaacson JS, Nicoll RA (1993) The uptake inhibitor L-trans-PDC enhances responses to glutamate but fails to alter the kinetics of excitatory synaptic currents in the hippocampus. J Neurophysiol 70:2187-2191.

Kanai Y, Hediger MA (1992) Primary structure and functional characterization of a high-affinity glutamate transporter. Nature 360:467-471.

Kanai Y, Nussberger S, Romero MM, Boron WF, Hebert SC, Hediger MA (1995) Electrogenic properties of the epithelial and neuronal high affinity glutamate transporter. J Biol Chem 270:16561-16568.

Kirino T (1993) Presynaptic terminals in hippocampal gliosis following transient ischemia in the Mongolian gerbil. Prog Brain Res 96:261-270.

Klockner U, Storck T, Conradt M, Stoffel W (1995) Functional properties and substrate specificity of the cloned L-glutamate/L-aspartate transporter GLAST-1 from rat brain expressed in Xenopus oocytes. J Neurosci 14:5759-5765.

Konnerth A, Obaid AL, Salzberg BM (1987) Optical recording of electrical activity form parallel fibres and other cell types in skate cerebellar slices in vitro. J Physiol (Lond) 393:681-702.
Kosaka T, Hama K (1986) Three-dimensional structure of astrocytes in the rat dentate gyrus. J Comp Neurol 249:242-260.

Lehre KP, Levy LM, Ottersen OP, Storm-Mathisen J, Danbolt NC (1995) Differential expression of two glial glutamate transporters in the rat brain: quantitative and immunocytochemical observations. J Neurosci 15:1835-1853.

Lester HA, Cao Y, Mager S (1996) Listening to neurotransmitter transporters. Neuron 17:807-810.

Lester RAJ, Clements JD, Westbrook GL, Jahr CE (1990) Channel kinetics determine the time course of NMDA receptor-mediated synaptic currents. Nature 346:565-567.

Luscher C, Malenka RC, Nicoll RA (1998) Monitoring glutamate release during LTP with glial transporter currents. Neuron 21:435-441.

Malandro MS, Kilberg MS (1996) Molecular biology of mammalian amino acid transporters. Annu Rev Biochem 65:305-336.

Martin DL (1995) The role of glia in the inactivation of neurotransmitters. In: Neuroglia (Kettenmann H, Ransom BR, eds), pp 732-745. Oxford: Oxford UP.

McKhann II GM, D'Ambrosio R, Janigro D (1997) Heterogeneity of astrocyte resting membrane potentials and intercellular coupling revealed by whole-cell and gramicidin perforated patch recordings from cultured neocortical and hippocampal slice astrocytes. J Neurosci 17:6850-6863.

Mennerick S, Zorumski CF (1994) Glial contributions to excitatory neurotransmission in cultured hippocampal cells. Nature 368:59-62.

Mennerick S, Zorumski CF (1995) Presynaptic influence on the time course of fast excitatory synaptic currents in cultured hippocampal cells. J Neurosci 15:3178-3192.

Mennerick S, Dhond RP, Benz A, Xu W, Rothstein JD, Danbolt NC, Isenberg KE, Zorumski CF (1998) Neuronal expression of the glutamate transporter GLT-1 in hippocampal microcultures. J Neurosci 18:4490-4499.

Nagano S, Kwak S, Kanazawa I (1997) EAAT4, a glutamate transporter with properties of a chloride channel, is predominantly localized in Purkinje cell dendrites, and forms parasagittal compartments in rat cerebellum. Neuroscience 78:929-933.

Otis TS, Wu Y-C, Trussell LO (1996) Delayed clearance of transmitter and the role of glutamate transporters at synapses with multiple release sites. J Neurosci 16:1634-1644.

Pines G, Danbolt NC, Bjoras M, Zhang Y, Bendahan A, Eide L, Koepsell H, Storm-Mathisen J, Seeberg E, Kanner BI (1992) Cloning and expression of a rat brain L-glutamate transporter. Nature 360:464-467.

Rothstein JD, Martin L, Levey AI, Dykes-Hoberg M, Jin L, Wu D, Nash N, Kuncl RW (1994) Localization of neuronal and glial glutamate transporters. Neuron 13:713-725.

Rothstein JD, Dykes-Hoberg M, Pardo CA, Bristol LA, Jin L, Kuncl RW, Kanai Y, Hediger MA, Wang Y, Schielke JP, Welty DF (1996) Knockout of glutamate transporters reveals a major role for astroglial transport in excitotoxicity and clearance of glutamate. Neuron 16:675-686.

Sarantis M, Ballerini L, Miller B, Silver RA, Edwards M, Attwell D (1993) Glutamate uptake from the synaptic cleft does not shape the decay of the non-NMDA component of the synaptic current. Neuron 11:541-549.

Storck T, Schulte S, Hofmann K, Stoffel W (1992) Structure, expression and functional analysis of a $\mathrm{Na}^{+}$dependent glutamate/aspartate transporter from rat brain. Proc Natl Acad Sci USA 89:10955-10959.

Tanaka K, Watase K, Manabe T, Yamada K, Watanabe M, Takahashi K, Iwama H, Nishikawa T, Ichihara N, Kikuchi T, Okuyama S, Kawashima N, Hori S, Takimoto M, Wada K (1996) Epilepsy and exacerbation of brain injury in mice lacking the glutamate transporter GLT-1. Science 276:1699-1702.

Wadiche JI, Arriza JL, Amara SG, Kavanaugh MP (1995) Kinetics of a human glutamate transporter. Neuron 14:1019-1027.

Zerangue N, Kavanaugh MP (1996) Flux coupling in a neuronal glutamate transporter. Nature 383:634-637. 